\title{
The bovine dilated cardiomyopathy locus maps to a 1.0-Mb interval on chromosome 18
}

\author{
Marta Owczarek-Lipska - Catherine Denis · \\ André Eggen - Tosso Leeb - Horst Posthaus · \\ Gaudenz Dolf · Martin H. Braunschweig
}

Received: 12 November 2008/ Accepted: 30 December 2008/Published online: 14 February 2009

(C) Springer Science+Business Media, LLC 2009

\begin{abstract}
Cardiomyopathies are myocardial diseases that lead to cardiac dysfunction, heart failure, arrhythmia, and sudden death. In human medicine, cardiomyopathies frequently warrant heart transplantation in children and adults. Bovine dilated cardiomyopathy (BDCMP) is a heart muscle disorder that has been observed during the last 30 years in cattle of Holstein-Friesian origin. In Switzerland BDCMP affects Swiss Fleckvieh and Red Holstein breeds. BDCMP is characterized by a cardiac enlargement with ventricular remodeling and chamber dilatation. The common symptoms in affected animals are subacute subcutaneous edema, congestion of the jugular veins, and tachycardia with gallop rhythm. A cardiomegaly with dilatation and hypertrophy of all heart chambers, myocardial degeneration, and fibrosis are typical postmortem findings. It was shown that all BDCMP cases reported worldwide traced back to a red factor-carrying HolsteinFriesian bull, ABC Reflection Sovereign. An autosomal
\end{abstract}

Electronic supplementary material The online version of this article (doi:10.1007/s00335-009-9171-z) contains supplementary material, which is available to authorized users.

M. Owczarek-Lipska ( $\square)$ · T. Leeb · G. Dolf ·

M. H. Braunschweig

Institute of Genetics, Vetsuisse Faculty, University of Berne, Bremgartenstrasse 109a, 3001 Berne, Switzerland

e-mail: marta.owczarek@itz.unibe.ch

C. Denis · A. Eggen

Laboratoire de Génétique biochimique et de Cytogénétique, UR339, INRA-CRJ, 78350 Jouy-en-Josas, France

H. Posthaus

Institute of Animal Pathology, Vetsuisse Faculty, University of Berne, 3001 Berne, Switzerland recessive mode of inheritance was proposed for BDCMP. Recently, the disease locus was mapped to a $6.7-\mathrm{Mb}$ interval MSBDCMP06-BMS2785 on bovine $\mathrm{Chr} 18$ (BTA18). In the present study the BDCMP locus was fine mapped by using a combined strategy of homozygosity mapping and association study. A BAC contig of $2.9 \mathrm{Mb}$ encompassing the crucial interval was constructed to establish the correct marker order on BTA18. We show that the disease locus is located in a gene-rich interval of $1.0 \mathrm{Mb}$ and is flanked by the microsatellite markers DIK3006 and MSBDCMP51.

\section{Introduction}

Cardiomyopathies are diseases of the myocardium associated with cardiac dysfunction. In human medicine they are classified as dilated cardiomyopathy, hypertrophic cardiomyopathy, restrictive cardiomyopathy, and arrhythmogenic right ventricular cardiomyopathy (Richardson et al. 1996). Many conditions characteristic for one form of a cardiomyopathy may progress to another condition. Cardiomyopathy frequently results in heart failure, arrhythmia, and sudden death. The histologic findings in such a dysfunctional myocardium are nonspecific, with hypertrophic myocytes, cellular necrosis, and fibrosis.

Bovine dilated cardiomyopathy (BDCMP) is a primary disease of the heart muscle observed worldwide in Holstein-Friesian cattle during the last 30 years. In Switzerland the introduction of Red Holstein genetics into the Simmental population began in 1968. These crosses are referred to as Fleckvieh and form a section apart from Simmental and Red Holstein. BDCMP affects Fleckvieh and Red Holstein cattle but not the Simmental cattle of the 
Swiss Fleckvieh breed (Graber and Martig 1993; Martig 1992; Martig et al. 1982). The disease is characterized by a global cardiac enlargement and ventricular remodeling leading to a chamber dilatation with decreased wall thickness and a diminution in systolic function. A subacute subcutaneous edema within the brisket and to a lesser extent in the mandibular and the ventral abdominal region is usually observed. Moreover, an increased pulse rate, up to 140 beats/min, tachycardia with gallop rhythm of the heart, and congestion of the jugular veins are common in affected animals. The typical age at onset of BDCMP is between 2 and 4 years but BDCMP was also diagnosed in calves younger than 2 months and in cows older than 8 years (Graber and Martig 1993; Martig 1992; Martig and Reusser 1988). Although BDCMP is a slowly progressive terminal disease, distinct clinical symptoms develop within a few days or weeks and all affected animals die or have to be culled shortly after disease onset (Bradley et al. 1991; Danzl 1995; Kümper and Bahnemann 1992; Martig and Tschudi 1985; Martig et al. 1982; McLennan and Kelly 1990; Sonoda et al. 1982; Tontis et al. 1990). Initially, cardiomyocyte necrosis in all parts of the heart followed by transmural fibrosis often occurs (König et al. 1990). First, the left ventricle fails to compensate, leading to pulmonary hypertension, followed by hypertrophy of the right ventricle, which finally causes the clinically manifest right heart failure (Lobsiger et al. 1985; Martig and Tschudi 1985). BDCMP can be easily misdiagnosed as traumatic pericarditis or valvular endocarditis (Martig et al. 1982). The pathologic findings are cardiomegaly with an extensive dilatation and hypertrophy of all sections of the myocardium, as well as myocardial fibrosis, partially accompanied by myofibrillar degeneration. To date, there is no treatment known for BDCMP.

All reported cases in Canada (Baird et al. 1986), Japan (Satoh 1988), and Switzerland (Martig and Reusser 1988; Martig et al. 1982) were traced back to a red factor-carrying Holstein-Friesian bull, ABC Reflection Sovereign, which strongly suggests a hereditary nature of the disease. A segregation analysis based on an experimental BDCMP pedigree confirmed the genetic basis of an autosomal recessive mode of inheritance for the disease (Dolf et al. 1998). Recently, BDCMP cases were reported in Denmark (Leifsson and Agerholm 2004). BDCMP is periodically diagnosed in slaughterhouses in Switzerland, but the frequency of the mutant allele responsible for the disease remains unknown.

The locus for bovine dilated cardiomyopathy was mapped to Chr 18 (BTA18) in the MSBDCMP06-BMS2785 interval (Guziewicz et al. 2007). In present study we fine-mapped the BDCMP locus to a $1.0-\mathrm{Mb}$ interval by a combined homozygosity mapping and association-based strategy.

\section{Material and methods}

\section{BAC libraries}

Two bovine BAC libraries were explored to establish a BAC contig around the BDCMP locus on BTA18 (CHORI-240: bovine BAC library, Children's Hospital Oakland Research Institute, USA and the INRA bovine BAC library) Eggen et al. 2001). CHORI BAC clones were selected based on the contig information of BTA18 which was released together with the bovine genome assembly Btau 3.1 (http://www. hgsc.bcm.tmc.edu/projects/bovine/). The INRA BAC library was PCR-screened to identify the corresponding BAC clones. In addition, we used the web-based BAC contig information tool (http://genome.jouy.inra.fr/WebAGCoL/ CarteBovinGenet/WebFPC/).

\section{DNA extraction from BAC clones}

BAC DNA was isolated according to the rapid alkaline lysis miniprep method developed by the Children's Hospital Oakland Research Institute (http://bacpac.chori.org/ bacpacmini.htm).

\section{BAC clones screening}

BAC DNA from clones of the CHORI BAC library was tested by PCR. BAC DNA pools from the INRA BAC library were first PCR-screened and subsequently positive clones were examined by PCR-based strategy for the presence of specific markers and genes. The PCR amplification mixture contained $1.5 \mu \mathrm{l}$ of BAC DNA (25 ng), $0.4 \mu \mathrm{l}$ of the primer $(10 \mathrm{pmol} / \mu \mathrm{l}$, forward and reverse, respectively), $0.2 \mu \mathrm{l}$ of dNTPs mix $(5 \mathrm{mM}), 1 \mu \mathrm{l}$ of $10 \times$ PCR buffer, and $0.05 \mu \mathrm{l}$ of AmpliTaqGold polymerase $(5 \mathrm{U} / \mu \mathrm{l})$, and deionized water was added to a final volume of $10 \mu \mathrm{l}$ (Applied Biosystems, Rotkreuz, Switzerland). The amplification was performed with an initial denaturation step at $95^{\circ} \mathrm{C}$ for $20 \mathrm{~min}$, followed by 30 cycles with a denaturation step at $94^{\circ} \mathrm{C}$ for $30 \mathrm{sec}$, an annealing step at $60^{\circ} \mathrm{C}$ for $1 \mathrm{~min}$, an extension step at $72^{\circ} \mathrm{C}$ for $2 \mathrm{~min}$, and a final extension phase at $72^{\circ} \mathrm{C}$ for $10 \mathrm{~min}$.

The amplicons were separated on $1 \%$ agarose gels, stained with ethidium bromide, and visualized under UV light. Primer pairs were designed with the PRIMER3 software and are listed in Supplementary Table 1.

To screen BAC clones, 20 microsatellite markers, 7 PCR products with known location on BTA18, and 27 PCR products of orthologous genes located on human counterpart Chr 19 (HSA19) were used. 


\section{DNA extraction}

DNA from 49 blood samples was isolated using Chelex ${ }^{\circledR}$ 100 (BioRad, Reinach BL, Switzerland) or a High Pure PCR Template Purification Kit (Roche Diagnostics, Rotkreuz, Switzerland). DNA from 304 formalin-fixed and thereafter paraffin-embedded myocardial samples from BDCMP-affected animals was isolated and purified using a DNeasy Blood \& Tissue Kit (Qiagen, Hombrechtikon, Switzerland).

Genotyping with microsatellite markers

In the present study, samples from 353 animals were genotyped for 24 microsatellite markers (MS) in the interval between MSBDCMPO6 and BMS2785, whereof 9 were selected from publically available data and 15 represent newly developed markers taken from the bovine genome sequence assembly Btau 4.0 (Supplementary Table 2).

The volume of a multiplex amplification reaction was $10 \mu \mathrm{l}$ and contained $2 \mu \mathrm{l}$ of DNA ( $20 \mathrm{ng}$ ), $0.2 \mu \mathrm{l}$ of each primer (10 pmol/ $\mu \mathrm{l}$, forward and reverse, respectively), $5 \mu \mathrm{l}$ of Multiplex PCR Master Mix (Qiagen), and $1 \mu \mathrm{l}$ of $5 \times$ Q-solution. Water was added to reach the final volume. Most of the multiplex reactions contained a combination of five different primer pairs. The PCR was conducted under the following conditions: initial denaturation step at $95^{\circ} \mathrm{C}$ for $15 \mathrm{~min}, 30$ cycles with a denaturation step at $94^{\circ} \mathrm{C}$ for $30 \mathrm{sec}$, an annealing step at $60^{\circ} \mathrm{C}$ for $1 \mathrm{~min}$, and an extension step at $72^{\circ} \mathrm{C}$ for $45 \mathrm{sec}$. The final extension step was carried out for $30 \mathrm{~min}$ at $60^{\circ} \mathrm{C}$. PCR products were initially examined on $2 \%$ agarose gels and, subsequently, $1 \mu \mathrm{l}$ of each PCR was combined with $10 \mu \mathrm{l}$ of genotyping mix $(980 \mu \mathrm{l}$ of HiDi formamide and $20 \mu \mathrm{l}$ of GeneScan $^{\mathrm{TM}}-500$ LIZ $^{\mathrm{TM}}$ Size Standard, Applied Biosystems). After a 2-min denaturation at $95^{\circ} \mathrm{C}$ and chilling on ice, samples were genotyped on an ABI 3730 capillary sequencer (Applied Biosystems). The data were processed using the GeneMapper ${ }^{\circledR}$ software ver. 4.0 (Applied Biosystems).

\section{SNP genotyping}

Annotated SNPs (http://www.ncbi.nlm.nih.gov/sites/entrez? $\mathrm{db}=\mathrm{snp}$ ) were selected according to their location within the interval of interest on BTA18. Additional SNP markers were developed in order to increase the marker density in the critical interval. These SNPs in the $1.0-\mathrm{Mb}$ region between microsatellites markers DIK3006 and MSBDCMP51 flanked by the distal genes $A P O E$ and NPASI were genotyped using a panel of 18 BDCMP-affected animals and 18 controls. In total, 38 PCR products were obtained according to standard protocols (Supplementary Table 3 ). These 38 PCR products each contained one to eight SNPs resulting in a total of 123 SNPs. Amplicons were purified with rAPid alkaline phosphatase (Roche) and exounuclease I (N.E.B., Bioconcept, Allschwil, Switzerland). Both strands were directly sequenced on an ABI 3730 capillary sequencer (Applied Biosystem) using the BigDye ${ }^{\circledR}$ Terminator Cycling Kit (Applied Biosystems). Sequencing data were analyzed using the Sequencher 4.8 software (Gene Codes Corporation, Ann Arbor, MI, USA).

Statistical analysis

The statistical analysis including genotypes of 123 SNP markers was initially performed with SAS Genetics 9.1.3 software (SAS Institute Inc., Cary, NC, USA) using the procedure allele. The established minor allele frequency (MAF) was 20\%. In total, 86 SNP markers were analyzed with the SAS Genetics 9.1.3 software procedure casecontrol and with the Haploview 4.0 software (Barrett et al. 2005) (http://www.broad.mit.edu/mpg/haploview/download.php). A permutation test was carried out with 1000 permutations and the single marker procedure was selected.

\section{Results and discussion}

\section{BAC contig construction}

The comparison between the cattle and the human maps revealed that the critical region on bovine $\mathrm{Chr} 18$ (BTA18q) is homologous to human Chr 19 (HAS19) (Guziewicz 2004). Recently, Guziewicz et al. (2007) demonstrated in an experimental pedigree segregating for BDCMP that the disease locus maps to a $6.7-\mathrm{Mb}$ region encompassed by MS markers MSBDCMPO6 and BMS2785 on BTA18 (Btau 3.1). This interval corresponds now to $5.6 \mathrm{Mb}$ in the bovine genome sequence assembly Btau 4.0. The publicly available BAC contig listing of bovine CHORI BACs was initially used to build the contig around the BDCMP locus. Although the information of the overlapping genomic sequence was indicated in the BAC contig provided by the bovine genome project, several overlaps could not be verified by PCR-based STS content mapping. Therefore, additional BAC clones were introduced from the INRA bovine BAC library in the attempt to close the remaining gaps. The combination of clones from the two BAC libraries allowed us to construct a 2.9-Mb BAC contig spanning the interval between MS markers MSBDCMP22 and BMS2785 (Fig. 1b). The minimal tiling path of this contig consists of 23 tiled BAC clones. We found that the gene order on a chromosomal segment 
between positions 51.11 and $52.12 \mathrm{Mb}$ was inverted in Btau 3.1 as compared to our BAC contig and the human counterpart HSA19. This finding was then fully confirmed in the recent cattle genome assembly (Btau 4.0), where the gene order in the critical interval is in agreement with that in our BAC contig and human Chr 19. The BAC contig is an excellent tool toward fine mapping the critical interval, to map the DNA markers, and to explore the still existing and numerous DNA sequence gaps in the present version of the bovine genome.

\section{Homozygosity mapping of the BDCMP locus}

This approach aims to identify the autosomal recessive disease-causing locus by virtue of the fact that the adjacent DNA sequence is homozygous by descent. It is evident that all BDCMP-affected animals in the Swiss Fleckvieh population trace back to the Canadian bull ABC Reflection Sovereign (Dolf et al. 1998). Therefore, alleles adjacent to the BDCMP locus are considered identical by descent and share extended haplotypes. In an initial step 25 BDCMPaffected and 24 unaffected Fleckvieh animals were genotyped with nine MS markers randomly distributed within the interval between MS markers MSBDCMP06 and $B M S 2785$, previously defined by Guziewicz et al. (2007). All the affected animals were homozygous at the markers MSBDCMP22, DIK3006, DIK3014, MSBDCMP25, and RME01 supporting the hypothesized identity by descent. Chromosomes were considered recombinant for an individual that is heterozygous for markers located distal of the interval MSBDCMP06-BMS2785 and homozygous for adjacent markers located within this interval. We found 11 presumably recombinant chromosomes on the centromeric site and 9 on the telomeric site of the interval MSBDCMP06-BMS2785. This homozygosity mapping narrowed the critical interval down to about $4.1 \mathrm{Mb}$ between markers MSBDCMP06 and MSBDCMP51. An additional 15 evenly distributed MS markers were selected within the interval MSBDCMP06-BMS2785 based on the bovine genome sequence assembly Btau 4.0. The markers were chosen so that they were evenly distributed in the interval. DNA from 304 paraffin-embedded heart tissue samples of cattle with a positive BDCMP diagnosis, including some cases with uncertain diagnoses, were available for genotyping. The advantage of these paraffinembedded samples is that the BDCMP diagnosis can be reevaluated based on histologic criteria by an experienced pathologist. Furthermore, these paraffin-embedded samples were collected during a period of about 20 years at two different locations in Switzerland and the BDCMP animals were originally diagnosed by different pathologists. The reevaluation is important as BDCMP can be easily misdiagnosed. We genotyped all these samples with $24 \mathrm{MS}$ markers in the interval MSBDCMP06-BMS2785. The identification of an additional 3 and 18 recombinant chromosomes from the centromeric and telomeric side, respectively, allowed us to reduce the critical interval from 4.1 to $1.0 \mathrm{Mb}$. The current $1.0-\mathrm{Mb}$ interval is flanked by MS markers DIK3006 and MSBDCMP51 (Fig. 1a). It is striking that the BDCMP-affected animals, previously assumed to be unrelated, are all homozygous for the markers that were also homozygous in the BDCMP animals in the experimental pedigree. This finding strongly indicates that indeed all affected animals are descendents from a single founder animal.

\section{Association study}

In this study we combined homozygosity mapping with an association-based strategy to further fine map the BDCMP locus in the interval between MS DIK3006 and MSBDCMP51 (Fig. 1a). We included on each distal site of this interval additional SNP markers located in $A P O E$ and NPAS1 genes, respectively. The association study was performed including these markers and indicates that the region outside of DIK3006 and MSBDCMP51 is not associated with BDCMP. In this interval a total of 36 PCR products containing one to eight SNP markers were genotyped by means of DNA sequencing for 18 BDCMPaffected and 18 unaffected animals. In addition, two families comprising eight individuals from the experimental pedigree were included in the genotyping procedure to deduce the disease haplotype. BAC clones were tested by PCR to map the SNP markers to our BAC contig. However, one of these PCR products containing six SNPs (Supplementary Table 3) was thereafter excluded from the association study because we could not amplify it on any of the BAC clones. This emphasizes that the BAC contig can be a useful tool in the fine mapping of disease genes.

The association study was initially performed using SAS Genetics software. A minimal MAF of $20 \%$ was applied in the analysis resulting in the exclusion of 31 SNP markers. The SNP in NPAS1 gene was therefore excluded from the final analysis and is not presented in Fig. 1c. The genotypes of 86 remaining SNP markers were thereafter analyzed using the Haploview 4.0 software. The best observed $\chi^{2}$ was 60.4 and the best permutation $\chi^{2}(1000$ permutations, single marker) was 21.4. The analyses revealed significant association between markers and disease over the whole interval DIK3006-MSBDCMP51 and showed three highly associated regions (Fig. 1c).

The human HSA19 counterpart of this 1.0-Mb interval corresponds to a region between 50.50 and $52.11 \mathrm{Mb}$ (Build 36.6). This is a gene-rich region and contains 47 genes and 18 annotated hypothetical loci. In the mouse this region was found on Chr 7 (MMU7) between 17.07 and 
19.99 $\mathrm{Mb}$ (Build 37.1) but the gene order is inverted compared to that in human and cattle.

An in silico evaluation showed that among 47 genes on HSA19, CKM, VASP, PPP1R13L, DMPK, and AP2S1 genes are highly expressed in the cardiovascular system; moderately expressed are the GIPR, PNMAL2, and GRLF1 genes, and GPR4, IGFL3, HIF3A, PRKD2, STRN4, and FKRP are reported to be expressed in the heart (http:// www.dsi.univ-paris5.fr/genatlas/, http://www.ncbi.nlm.nih. gov/). An association between two of these genes, the fukutin-related protein gene $(F K R P)$ and the vasodilatorstimulated phosphoprotein gene (VASP), and dilated cardiomyopathy was reported. Müller et al. (2005) found evidence that dilated cardiomyopathy in human may be associated with a C826A mutation in the FKRP gene. Although mutations in this gene are mostly responsible for limb-girdle muscular dystrophy (LGMD2I), they described three siblings showing the C826A substitution and an unusual phenotype with dilated cardiomyopathy and without changes in skeletal muscles. Our preliminary results indicate that there are no differences in the FKRP sequence between BDCMP-affected and unaffected animals. Eigenthaler et al. (2003) described that a disruption of cardiac Ena-VASP protein in mice may play a crucial role in intercalated disk function at the interface between cardiac myocytes and thereby might cause dilated cardiomyopathy. However, to our knowledge no mutation in the $V A S P$ gene in any species has yet been reported to be associated with cardiomyopathy.

In conclusion, using the combined strategy of homozygosity mapping and association study, we were able to fine map the BDCMP locus to a region of about $1.0 \mathrm{Mb}$. At

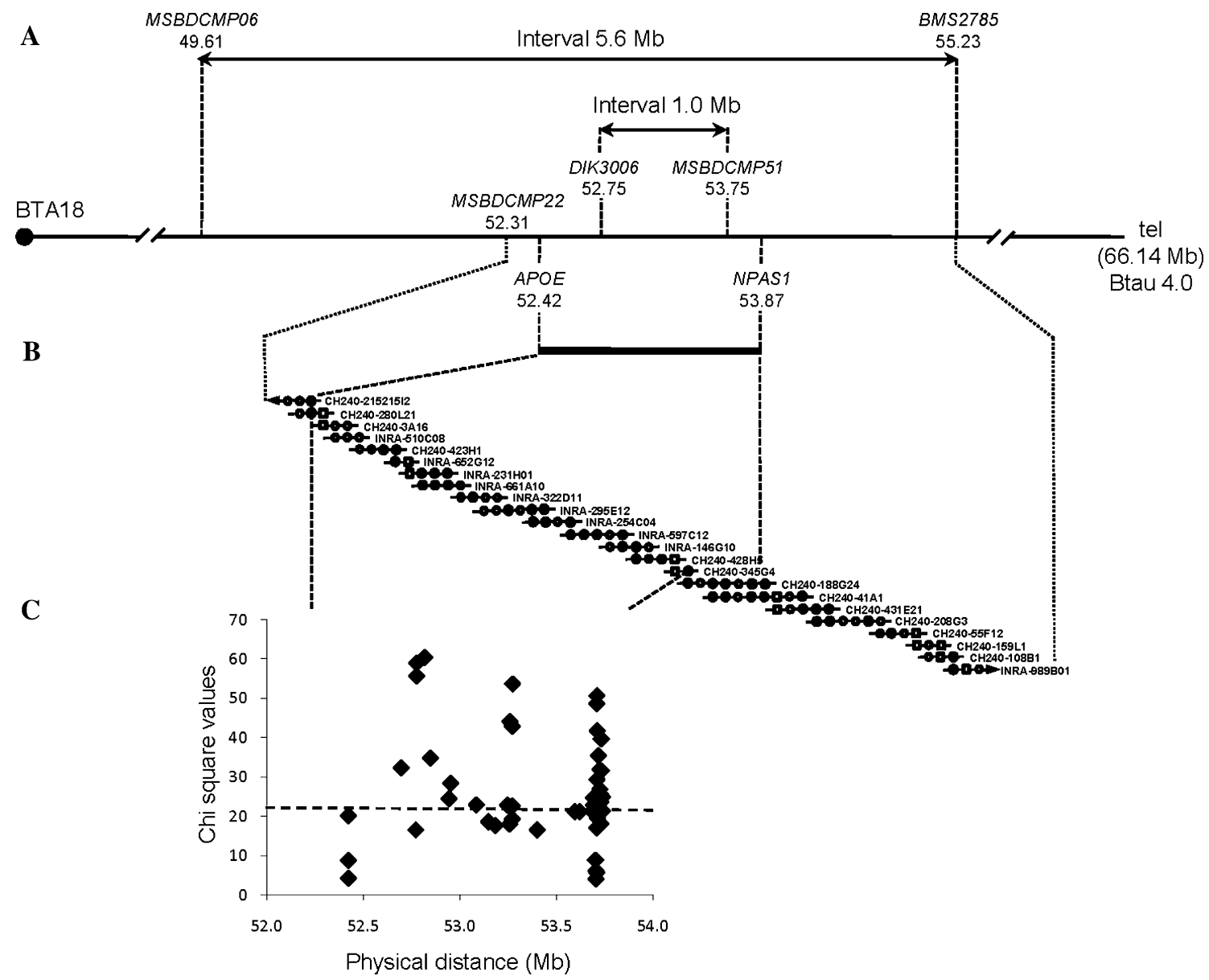

Fig. 1 Genetic mapping of the BDCMP locus. a Initial interval defined to $6.7 \mathrm{Mb}$ between MS markers MSBDCMP06 and BMS2785 based on Btau 3.1 corresponds to a physical distance of $5.6 \mathrm{Mb}$ on Btau 4.0. Current interval of $1.0 \mathrm{Mb}$ is flanked by MS markers $D I K 3006$ and MSBDCMP51. The association study was performed in the $1.0-\mathrm{Mb}$ interval surrounded by the distal genes APOE and NPAS1. b BAC contig of $2.9 \mathrm{Mb}$ between MS markers MSBDCMP22 and $B M S 2785$ composed of 23 BAC clones. Open circles, filled circles, and open squares indicate MS markers, genes, and PCR products with known location on BTA18, respectively. $\mathbf{c}$ Schematic presentation of the association analysis (Haploview 4.0 software). The best observed $\chi^{2}$ was 60.44 . The dashed line indicates the significance threshold obtained by permutation testing (best permutation $\chi^{2}$ was $21.45,1000$ permutations). $\chi^{2}$ values are plotted against the physical distance on BTA18. In total, 86 SNP markers with MAF above $20 \%$ were analyzed 
present we are screening recombinant animals with additional SNP markers to further narrow down the interval. In addition, we will further investigate those genes that are expressed in the cardiovascular system. It is still possible that genes with not yet known function as well as regulatory mutations or chromosomal rearrangements may play an important role in the development of BDCMP.

Acknowledgments The authors thank Brigitta Colomb for technical support. This research was supported by the Swiss National Foundation (Project 3100AG-108230).

\section{References}

Baird JD, Maxie MC, Kennedy BW, Harris DJ (1986) Dilated (congestive) cardiomyopathy in Holstein cattle in Canada, Genetic analysis of 25 cases. In: Proceedings of the 14th World Congress on Diseases of Cattle, 26-29 August 1986, Dublin, Ireland, pp 89-94

Barrett JC, Fry B, Maller J, Daly MJ (2005) Haploview: analysis and visualization of LD and haplotype maps. Bioinformatics 21:263265

Bradley R, Jefferies AR, Jackson PG, Wijeratne WV (1991) Cardiomyopathy in adult Holstein Friesian cattle in Britain. J Comp Pathol 104:101-112

Danzl H (1995) Bovine Kardiomyopathie in Österreich. Wien Tieraerztl Monschr 82:16-23

Dolf G, Stricker C, Tontis A, Martig J, Gaillard C (1998) Evidence for autosomal recessive inheritance of a major gene for bovine dilated cardiomyopathy. J Anim Sci 76:1824-1829

Eggen A, Gautier M, Billaut A, Petit E, Hayes H et al (2001) Construction and characterization of a new bovine BAC library with four genome-equivalent coverage. Genet Sel Evol 33:534548

Eigenthaler M, Engelhardt S, Schinke B, Kobsar A, Schmitteckert E et al (2003) Disruption of cardiac Ena-VASP protein localization in intercalated disks causes dilated cardiomyopathy. Am J Physiol Heart Circ Physiol 285:2741-2784

Graber HU, Martig J (1993) Diagnosis of bovine cardiomyopathy by electrolyte and protein analysis. Zentralbl Veterinarmed A 40:690-696

Guziewicz KE (2004) Bovine dilated cardiomyopathy: evidence for a major gene on BTA18. Doctoral thesis, Dissertation ETH No. 15817, Swiss Federal Institute of Technology, Zurich
Guziewicz KE, Owczarek-Lipska M, Küffer J, Schelling C, Tontis A et al (2007) The locus for bovine cardiomyopathy maps to chromosome 18. Anim Genet 38:265-269

König F, Zwahlen R, Schaller J, Kämpfer U, Roth D et al (1990) Bovine cardiomyopathy, pathomorphogenic and biochemical studies in yearling steers. Schweiz Arch Tierheilkd 132:439-440

Kümper H, Bahnemann R (1992) [Myocardial fibrosis of cattle in Hesse]. Tierarztl Prax 20:254-258 [article in German]

Leifsson PS, Agerholm JS (2004) Familial occurrence of bovine dilated cardiomyopathy in Denmark. J Vet Med A Physiol Pathol Clin Med 51:332-335

Lobsiger C, Rossi GL,Tontis A, Luginbühl H (1985) [Study of pulmonary vascular lesions in cows with cardiomyopathy]. Schweiz Arch Tierheilkd 127:479-503 [article in French]

Martig J (1992) Bovine dilated cardiomyopathy. In: Proceedings of the 8th International Conference on Production Disease in Farm Animals, Berne, Switzerland, 24-27 August 1992

Martig J, Reusser HR (1988) Abstammungsanalysen von an Kardiomayopathie erkrankten Kühen. Proceedings of the 15th World Buiatrics Congress, 11-14 October 1988, Palma de Mallorca, Spain, pp 580-584

Martig J, Tschudi P (1985) [Further cases of cardiomyopathy in cattle]. Dtsch Tieraerztl Wocherschr 92:363-366 [article in German]

Martig J, Tschudi P, Perritaz C, Tontis A, Luginbühl H (1982) [Incidence of cardiac insufficiency in cattle. Preliminary report]. Schweiz Arch Tierheilkd 124:69-82 [article in German]

McLennan MW, Kelly WR (1990) Dilated (congestive) cardiomyopathy in a Friesian heifer. Aust Vet J 67:75-76

Müller T, Krasnianski M, Witthaut R, Deschauer M, Zierz S (2005) Dilated cardiomopathy may be an early sign of the C826A Fruktin-related protein mutation. Neuromuscul Disord 15:372376

Richardson P, McKenna W, Bristow M, Maisch B, Mautner B et al (1996) Report of the 1995 World Health Organization/International Society and Federation of Cardiology Task Force on the Definition and Classification of cardiomyopathies. Circulation 93:841-842

Satoh T (1988) Studies on the dilated cardiomyopathy in cattle. Bull Nippon Vet Zootech Coll 37:152-154

Sonoda M, Takahashi K, Kurosawa T, Matsukawa K, Chiyada Y (1982) Clinical and clinico-pathological studies on idiopathic congestive cardiomyopathy in cattle. Proceedings of the 12th World Congress on Diseases of Cattle, 7-10 September 1982, Amsterdam, The Netherlands, pp 1187-1191

Tontis A, Zwahlen R, Lobsiger C, Luginbühl H (1990) Pathology of bovine cardiomyopathy. Schweiz Arch Tierheilkd 132(3):105116 [article in German] 